\title{
Disruption of PTPS Gene Causing Pale Body Color and Lethal Phenotype in the Silkworm, Bombyx mori
}

\author{
Xiaoling Tong ${ }^{1,+}$, Pingfeng Liang ${ }^{1,+}$, Songyuan $\mathrm{Wu}^{1,+}$, Yuanhao $\mathrm{Li}^{1}{ }^{1}$, Liang Qiao ${ }^{1}{ }^{\mathbb{1}}$, Hai Hu ${ }^{1}$, \\ Zhonghuai Xiang ${ }^{1}$, Cheng $\mathrm{Lu}^{1, *}$ and Fangyin Dai ${ }^{1,2, *}$ \\ 1 State Key Laboratory of Silkworm Genome Biology, Key Laboratory of Sericulture Biology and Genetic \\ Breeding, Agricultural Ministry, College of Biotechnology, Southwest University, Chongqing 400715, China; \\ xltong@swu.edu.cn (X.T.); lpf230@163.com (P.L.); fly_jungle@163.com (S.W.); lyh.1860@163.com (Y.L.); \\ qiaoliangswu@163.com (L.Q.); huhaiswu@163.com (H.H.); xbxzh@swu.edu.cn (Z.X.) \\ 2 College of Biotechnology, Southwest University, Chongqing 400715, China \\ * Correspondence: lucheng@swu.edu.cn (C.L.); fydai@swu.edu.cn (F.D.); Tel.: +86-23-6825-0793 (C.L. \& F.D.) \\ + These authors contributed equally to this work.
}

Received: 13 February 2018; Accepted: 27 March 2018; Published: 29 March 2018

\begin{abstract}
Phenylketonuria (PKU) is an inborn error of metabolism caused by mutations in the phenylalanine hydroxylase $(P A H)$ gene or by defects in the tetrahydrobiopterin (BH4) synthesis pathway. Here, by positional cloning, we report that the 6-pyruvoyl-tetrahydropterin synthase (PTPS) gene, encoding a key enzyme of $\mathrm{BH} 4$ biosynthesis, is responsible for the $\mathrm{ll}^{\mathrm{c}}$ (albino $\mathrm{C}$ ) mutation that displays pale body color, head shaking, and eventually lethality after the first molting in silkworm. Compared to wild type, the $a l^{c}$ mutant produced more substrates (phenylalanine (Phe) and tyrosine (Tyr)) and generated less DOPA and dopamine. Application of 2,4-diamino-6-hydroxypyrimidine (DAHP) to block BH4 synthesis in the wild type effectively produced the $a^{c}$-like phenotype, while BH4 supplementation rescued the defective body color and lethal phenotype in both $a l^{c}$ and DAHP-treated individuals. The detection of gene expressions and metabolic substances after drugs treatments in $\mathrm{al}^{c}$ and normal individuals imply that silkworms and humans have a high similarity in the drugs metabolic features and the gene pathway related to $\mathrm{BH} 4$ and the dopamine biosynthesis. We propose that the $a l^{c}$ mutant could be used as an animal model for drug evaluation for BH4-deficient PKU.
\end{abstract}

Keywords: silkworm model; $a l^{c}$; PTPS; BH4; phenylketonuria

\section{Introduction}

Phenylketonuria (PKU; OMIM 261600) is an inherent disorder of metabolism predominantly caused by mutations in the phenylalanine hydroxylase $(P A H)$ gene, which results in the accumulation of phenylalanine (Phe). PAH associates with a native co-factor tetrahydrobiopterin (BH4) that hydroxylates Phe to tyrosine (Tyr) (Supplementary Figure S1A). Defects in either PAH or BH4 synthesis may result in hyperphenilalaninemia, which can cause intellectual disability when untreated [1].

Phenylketonuria may result in progressive irreversible neurological impairment during infancy and childhood when not detected and treated in a timely manner. The most common symptom is severe mental retardation, which is often coupled with a musty odor in their breath, skin, and urine, eczema, reduced melanin pigmentation, growth retardation, depressive and anxiety disorders, and epilepsy. The severity of the clinical phenotype is directly correlated to blood phenylalanine levels [2].

Neonatal screening for PKU is crucial, and because a PKU newborn shows no obvious clinical symptom, a continuous Phe-restricted diet can prevent intellectual disability [3,4]. However, in some BH4-defcienct PKU patients, this strategy has a limited role in impaired neuropsychological 
functioning, therefore, individuals are prone to depressive and anxiety problems [5]. The reason is that $\mathrm{BH} 4$ is not only a co-factor of PAH in the liver, but is also involved in cerebral neurotransmission by its co-factor and / or chaperone activities for Tyr hydroxylase (TH), tryptophan hydroxylase (TPH), and nitric oxide synthase [6-8] (Supplementary Figure S1B,C). Hence, more research is required to find a more thorough understanding and more efficient therapeutic strategy for the treatment of various clinical phenotypes in PKU patients.

The development of experimental animals is an important component in the study of human diseases. The silkworm, Bombyx mori, is a fully domesticated insect derived from the wild silkworm, $B$. mandarina, and has been used for silk production for about 5000 years [9]. The silkworm is dependent on humans for its survival. From the beginning of the 19th century, the silkworm has also been utilized as an animal model for scientific discovery in microbiology, physiology, and genetics. Through nearly 100 years of classical genetics, more than 600 mutant strains of the silkworm have been obtained and preserved in China and Japan. It was recently considered as a model organism for Lepidoptera, which is the second largest insect order, following the completion of its genome sequence. Today, the silkworm plays a role in three major areas: basic research, sericulture, and biotechnology [10].

Albino $\mathrm{C}\left(a l^{c}\right)$ is a spontaneous autosomal recessive larval stage mutant that is preserved in the silkworm gene bank of Southwest University, China. The body color of this mutant is normal dark-brown during the larval first instar, and turns pale after the first molting (Figure 1B). In contrast, the wild type (Dazao) shows normal pigmentation (Figure 1A). The mutants display head shaking behavior, and die within two days when untreated. Classic genetic research determined that this silkworm mutant was controlled by a single locus on chromosome 5 . In the present study, in order to identify the candidate gene for $a l^{c}$, fine mapping was performed. In addition, we propose a molecular mechanism underlying this particular phenotype and potential application of this disease model.
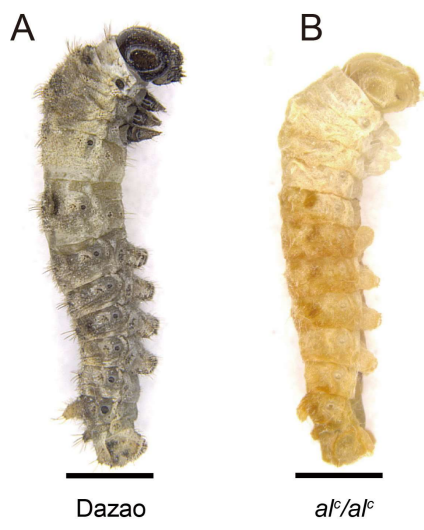

Figure 1. Phenotype of wild type (Dazao) and mutant albino $\mathrm{C}\left(\mathrm{al}^{\mathrm{c}}\right)$. At the beginning of the second instar, the $a^{\mathcal{c}}$ larvae showed a pale color in the epidermis and head (B) compared to the dark-brown color of the wild type Dazao (A). Scale bar: $1 \mathrm{~mm}$.

\section{Results}

\subsection{Fine Mapping and Gene Cloning}

To identify the responsible gene of the $\mathrm{al}^{c}$ mutant, genetic linkage analysis was performed. Owing to the lack of chromosomal recombination in female silkworms and the homozygous larval lethality of the albino $C$ mutant, we selected $F_{1}$ offspring from a cross between albino $C\left(+/ a l^{c}\right)$ female and Dazao $(+/+)$ male. Ten Dazao and ten albino $\mathrm{C}$ mutants of $\mathrm{BC}_{1} \mathrm{~F}$ (backcross female parent, first generation) were used in the cross, and the offspring were used for linkage analysis. In addition, $170 \mathrm{BC}_{1} \mathrm{M}$ (backcross male parent, first generation) progeny (only al ${ }^{c}$ individuals) from the male $\mathrm{F}_{1}$ backcrossed with albino $\mathrm{C}\left(+/ \mathrm{al} l^{c}\right)$, were used for recombination analysis. The $a l^{c}$ locus was mapped within $\sim 400 \mathrm{~kb}$ between markers C24 and D2 on Bm_nscaf 2674 (chromosome 5) (Figure 2A). This region encompasses 
18 predicted genes in the Silkworm Genome Database (http:/ / www.silkdb.org/silkdb/, SilkDB) [11]. The sequence of these predicted genes was used in a BLASTX search for their protein function in the non-redundant database (http:/ / www.ncbi.nlm.nih.gov/) (Table S1). Because of the defective body color and larval lethalithy of $a l^{c}$, the candidate genes that were involved in pigment metabolism and lethality were primarily considered in the screening of the offspring.
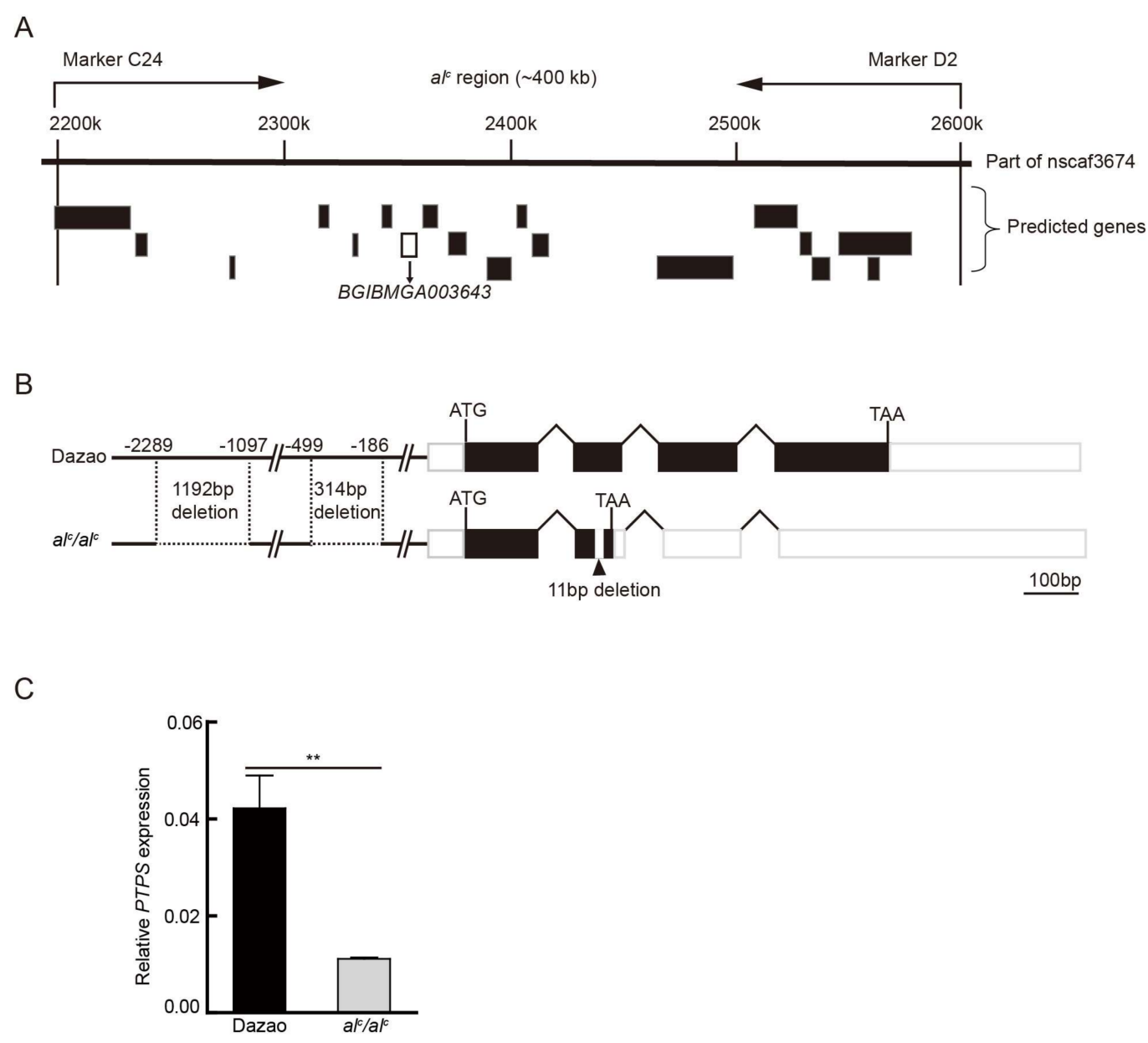

Figure 2. Positional cloning of the $a l^{\mathcal{C}}$ mutant. (A) Mapping of the $a l^{c}$ locus on linkage group 5 using $170 \mathrm{BC}_{1}$ (backcross, first generation) individuals. The $a l^{c}$ locus was narrowed down between the PCR markers C24 and D2, by approximately $400 \mathrm{~kb}$. Putative genes predicted by the Silkworm Genome Database are shown below the map, and BGIBMGA003643 is shown in the black box. (B) The genomic structure of BGIBMGA003643 in the wild type Dazao and $a l^{c}$ mutant. A horizontal line, white box, black box, and polygonal lines indicate the upstream regulatory region, UTRs, ORFs, and intronic regions, respectively. In $a l^{c}$, an 11-bp deletion in exon 2 results in a premature stop codon. In addition, 1192-bp and 314-bp deletions were detected in the $a l^{c}$ mutant in the upstream regulatory region. (C) Quantitative RT-PCR analysis of the relative expression levels of PTPS between Dazao and al $^{\mathcal{C}}$ on the first day of the second instar (student's $t$-test; $n=3,{ }^{* *} p<0.01$, data are expressed as the mean $\pm \mathrm{SD}$ ).

Based on the functional annotation of the 18 genes and the biological processes these may be involved in, the present study focused on one gene, BGIBMGA003643, which encodes 6-pyruvoyl-tetrahydropterin synthase (PTPS), a key enzyme in BH4 metabolism (Figure 2A). BH4 is an indispensable co-factor of $\mathrm{PAH}$ and $\mathrm{TH}$. BH4 deficiency can interrupt dopamine biosynthesis, subsequently affecting melanin pigmentation [12]. We cloned the cDNA (complementary DNA) of the PTPS gene in the wild type Dazao and $\mathrm{al}^{c}$. In the wild type, the transcript of PTPS was $1014 \mathrm{bp}$ in length, in which the open reading frame (ORF) was 504 bp in length and encoded a 167-amino acid 
protein. However, the transcript of $a l^{c}$ has several single nucleotide polymorphisms (SNPs) and an 11-bp deletion in exon 2. This deletion, which results in a frame shift and a premature stop codon, was identified (Figure 3B), and the deletion transcript apparently produces a functionally null protein. Moreover, the genomic DNA of PTPS was cloned in $a l^{c}$, and a 314-bp and 1192-bp deletion were detected upstream of the transcriptional start site (Figure 2B). The qRT-PCR analysis determined that the PTPS was significantly downregulated in $\mathrm{al}^{C}$ compared with that in Dazao at the beginning of the second instar (Figure 2C). PTPS was in complete linkage with the $a^{\mathcal{C}}$ mutant, and the gene has important related physiological functions and significant sequence defects in mutants. These observations prompted us to consider PTPS as a candidate gene of $a l^{c}$.

A

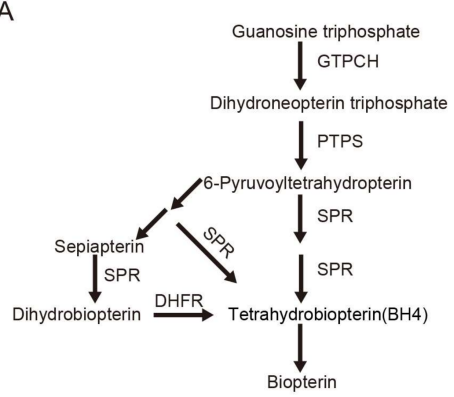

C

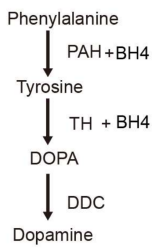

E

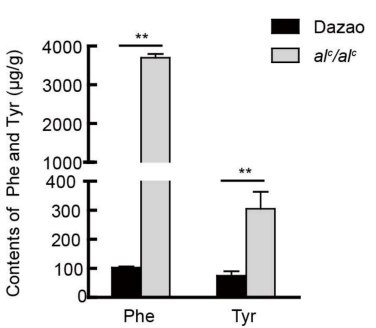

B

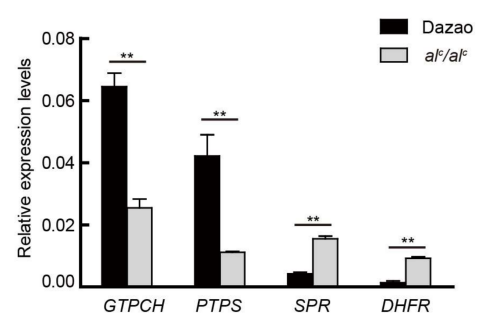

D

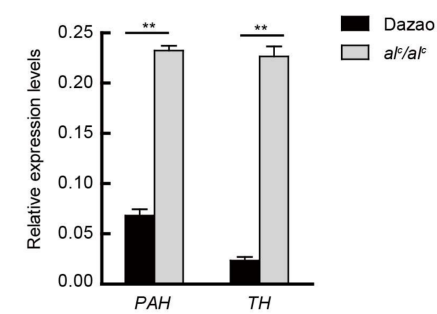

F

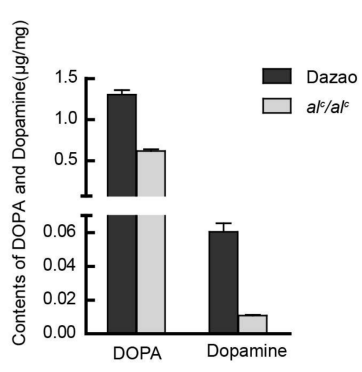

Figure 3. The comparison of related gene expression levels and material content in two pathways between Dazao and $a l^{c}$ mutant. (A) The biosynthesis pathway of tetrahydrobiopterin (BH4) and pteridines from guanosine triphosphate (GTP). GTPCH: GTP cyclohydrolase; PTPS: 6-pyruvoyl-tetrahydropterin synthase; DHFR: dihydrofolate reductase; SPR: sepiapterin reductase. (B) Quantitative RT-PCR analysis of key genes during the biosynthesis pathway of BH4 at the beginning of the second instar between Dazao and ll $^{\mathcal{c}}$ (student's $t$-test; $n=3,{ }^{* *} p<0.01$, data are expressed as the mean $\pm \mathrm{SD}$ ). (C) The biosynthesis pathway of melanin from phenylalanine. TH: tyrosine hydroxylase; PAH: Phenylalanine hydroxylase; DDC: DOPA decarboxylase. (D) Expression levels of the corresponding melanin genes between Dazao and $a l^{\mathcal{C}}$ at the beginning of the second instar (student's $t$-test; $n=3,{ }^{* *} p<0.01$, data are expressed as the mean $\pm \mathrm{SD}$ ). (E) Amino acid analysis in the melanin pathway between Dazao and $a l^{c}$ at the beginning of the second instar (student's $t$-test; $n=3$, ** $p<0.01$, data are expressed as the mean \pm SD). (F) Differences in DOPA and dopamine content at the beginning of the second instar between Dazao and $a l^{c}$ (student's $t$-test; $n=3,{ }^{* *} p<0.01$, data are expressed as the mean $\pm \mathrm{SD}$ ). 


\subsection{Expression Levels of Key Genes Involved in the BH4 Pathway}

BH4 is synthesized from guanosine triphosphate (GTP) through a cascade of three enzymes: GTP cyclohydrolase I (GTPCH), 6-pyruvoyl-tetrahydropterin synthase (PTPS), and sepiapterin reductase (SPR) (Figure 3A). PTPS is located upstream of the BH4 biosynthesis pathway. To clarify whether the inactivation of this enzyme affects the transcription levels of other key genes in this pathway, we investigated the expression levels of the GTPCH (AB439287), SPR (AK385894.1), and dihydrofolate ereductase (DHFR, JQ348842.1) genes in the Dazao and $a l^{c}$ strains by qRT-PCR at the beginning of the second instar. The expression level of GTPCH was significantly downregulated, whereas that of $S P R$ and DHFR were significantly upregulated in the $a l^{c}$ strain compared to that in wild type Dazao (Figure 3B).

PTPS dysfunction causes the abnormal accumulation of its substrate, dihydroneopterin triphosphate, which apparently inhibits the expression level of GTPCH. Moreover, a lack of substrates (6-pyruvoyltetrahydropterin and dihydrobiopterin) induces the upregulation of SPR and DHFR. On the other hand, wild type Dazao exhibited moderate levels of SPR and DHFR expression. The observed abnormal expression of these genes in the $\mathrm{BH} 4$ biosynthesis pathway is suggestive of a disruption in BH4 biosynthesis.

\subsection{The Expression Level of Key Genes and Content of Substances in the Melanin Metabolic Pathway}

$\mathrm{BH} 4$ is not only a co-factor of PAH in the liver, but is also involved in cerebral neurotransmission by its co-factor and/or chaperone activities for Tyr hydroxylase. A comparison of the expression of key genes involved in the biosynthesis pathway of $\mathrm{BH} 4$ between Dazao and alc indicate that $\mathrm{BH} 4$ synthesis is blocked. Further, assessment of the expression level of $P A H$ and $\mathrm{TH}$, which are important genes of the dopamine metabolic pathway (Figure 3C; Supplementary Figure S2) showed that the two genes were significantly up-regulated in $a^{c}$ relative to that in Dazao (Figure 3D). Furthermore, content of Phe and Tyr, which are catalyzed by $\mathrm{PAH}$ and $\mathrm{TH}$, respectively, were significantly higher in alc (36- and 4-fold higher, respectively) than that in Dazao (Figure 3E). In addition, the levels of DOPA and dopamine, which are the catalytic products of $\mathrm{TH}$ and important neurotransmitters and melanin precursors, were significantly lower (about 2.1- and 4.7-fold) in $a l^{c}$ compared to that in the Dazao strain, respectively (Figure $3 \mathrm{~F}$ ). The $\mathrm{ll}^{c}$ mutant produced more substrates (Phe and Tyr) and enzymes (PAH and $\mathrm{TH}$ ), but generated less DOPA and dopamine products; however, its deficiency in BH4 prevents them from synthesizing DOPA and dopamine. In addition, due to deficiencies in melanin precursors (DOPA and dopamine), the $\mathrm{al}^{c}$ mutants are incapable of exhibiting normal melanin pigmentation.

\subsection{Treatment Using a BH4 Inhibitor}

In the $a^{c}$ mutant, a disorder involving BH4 biosynthesis can lead to an excessive accumulation of Phe, thereby reducing DOPA and dopamine levels, ultimately resulting in albinism. The present study applied a BH4 biosynthesis inhibitor (2,4-diamino-6-hydroxypyrimidine, DAHP) to wild type strain to create this PAH deficiency phenotype, and to investigate the biochemical metabolic mechanism underlying this condition.

DAHP, a competitive inhibitor of GTPCH $[13,14]$, was administered to newborn larvae of the wild type strain N4, and their phenotypic features were assessed at the beginning of the second instar (first molting). Strain N4 treated with low basic sodium hydroxide solution was used as control. Individuals showing extremely similar features as that of the alc mutants were observed in the treatment group at day 1 of the second instar (Figure 4A). Simultaneously, we detected the expression level of key genes in the $\mathrm{BH} 4$ and melanin pathways of these individuals. The results show that the expression levels of these genes in the $a l^{c}$-like individuals were similar to that in the $a l^{c}$ mutants. The expression level of GTPCH and PTPS was significantly downregulated in the DAHP-treated group, whereas that of SPR, DHFR, $P A H$, and TH were upregulated (Figure 4B,C). These findings indicate that BH4 deficiency can lead to an albino phenotype, and the silkworm effectively responded to the drug treatment. 
A

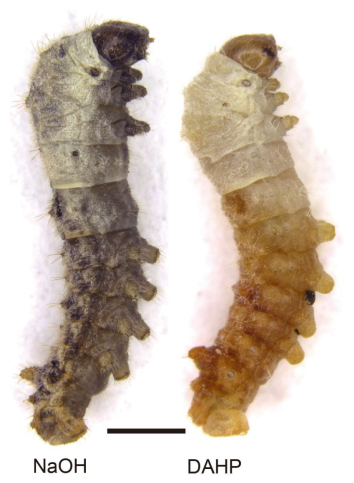

C
B

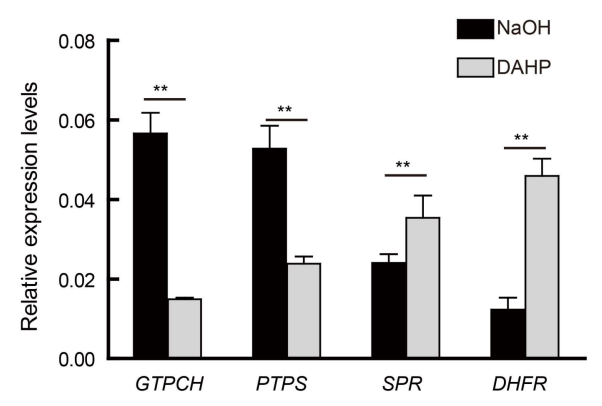

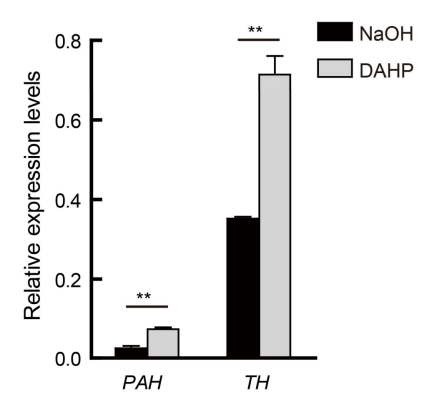

Figure 4. Oral administration of GTPCH inhibitor (2,4-diamino-6-hydroxypyrimidine (DAHP)). (A) The individual phenotype of treatment with GTPCH inhibitor, DAHP, and control group treatment with $\mathrm{NaOH}$ in the N4 strain at the beginning of the second instar. Scale bar: $1 \mathrm{~mm}$. (B) The relative expression level of corresponding genes during the biosynthesis pathway of BH4 between DAHP-treated and $\mathrm{NaOH}$-treated (control) at the beginning of the second instar (student's $t$-test; $n=3,{ }^{* *} p<0.01$, data are expressed as the mean $\pm \mathrm{SD}$ ). (C) Differences in expression level of $P A H$ and $T H$ between DAHP-treated and $\mathrm{NaOH}$-treated (control) at the beginning of the second instar (student's $t$-test; $n=3,{ }^{* *} p<0.01$, data are expressed as the mean $\pm \mathrm{SD}$ ).

\subsection{Treatment of BH4 Deficiency}

Disorders in BH4 biosynthesis can lead to hypopigmentation, head shaking, and death in silkworm. We next determined whether the lethality and pale body color of the natural mutation $\left(a l^{c}\right)$ and $a l^{c}$-like individuals induced by DAHP treatment could be rescued by BH4 supplement.

Newly hatched larvae of the $a l^{c}$ mutants and members of the DAHP treatment group received $30 \mathrm{mM}$ of $\mathrm{BH} 4$, and their phenotypes were monitored for the next two days. The same volume of $\mathrm{H}_{2} \mathrm{O}$ was given to the control group. The $\mathrm{BH} 4$ treatment group showed melanization compared to that in the control group (Figure 5A), and an increase in survival rate. Meanwhile, feeding dopamine to newly hatched larvae also converted the $a l^{c}$ phenotype to exhibit normal pigmentation and prolongation of their lifespan (data not shown).

In addition, we investigated the expression levels of key genes involved in melanin; $\mathrm{BH} 4$ pathways returned to their normal levels. In particular, the expression levels of $P A H$ and $T H$ significantly declined (Figure 5B). These results indicate that drug treatment can rescue the defective body color and significantly improve the survival ratio of diseased silkworm (alc and DAHP-treated individuals). 
A
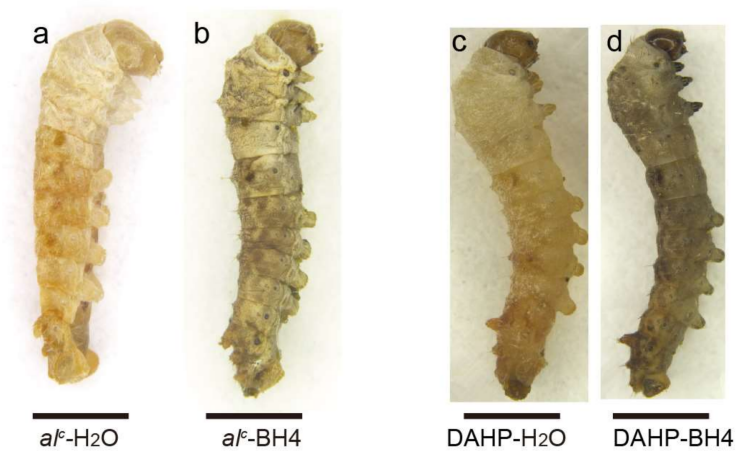

B

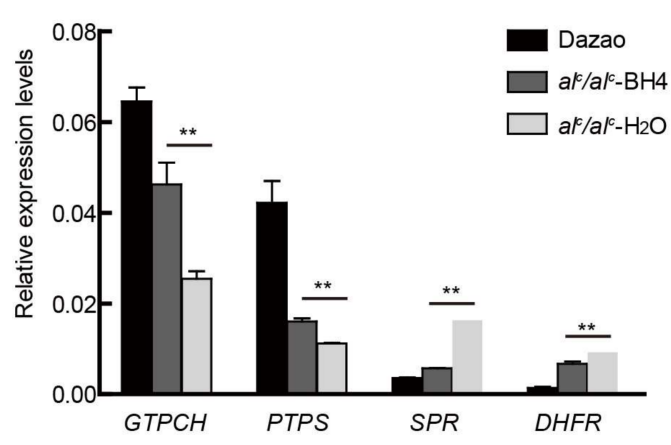

C

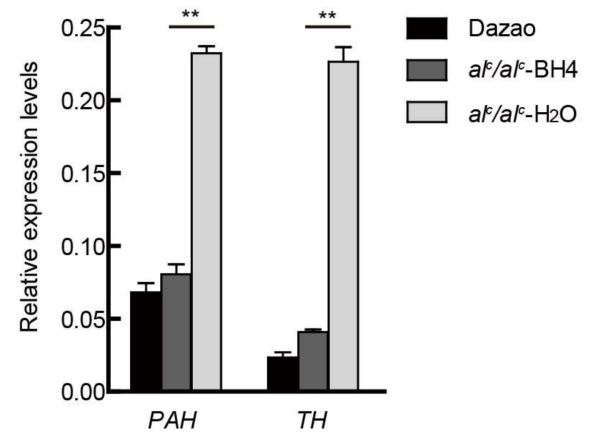

Figure 5. Rescue of the $a l^{c}$ larvae by BH4 administration. (A) Phenotype of $a l^{c}$ and DAHP-treated larvae in $30 \mathrm{mM}$ BH4 treatment on day 2 of the second instar. Scale bar: $1 \mathrm{~mm}$. (B) Expression differences in BH4 synthesis-related genes between Dazao and $a l^{\mathcal{C}}$ at day 2 of the second instar (student's $t$-test; $n=3$, ** $p<0.01$, data are expressed as the mean $\pm \mathrm{SD}$ ). (C) Expression levels of the melanin metabolism genes between wild type Dazao and $a l^{c}$ mutant at day 2 of the second instar (student's $t$-test; $n=3$, ** $p<0.01$, data are expressed as the mean $\pm \mathrm{SD}$ ).

\section{Discussion}

In the present study, the responsible gene for larval pigmentation, $a l^{c}$, was determined to encode PTPS, which is a key enzyme of BH4 biosynthesis. The $\mathrm{al}^{\mathrm{C}}$ mutant and wild type strain showed no observable differences during the embryonic and larval early instars. However, the body color of the $a l^{c}$ mutants switched from normal dark brown to pale at the beginning of the second instar. Furthermore, the $a l^{c}$ mutants showed excessive accumulation of Phe and deficiencies in DOPA and dopamine for 
cuticular pigmentation, thereby leading to an insufficient supply of $N$-acetyl dopamine (NADA) and $N$ - $\beta$-alanyldopamine (NBAD) for mouthparts sclerotization and larvae death after the first molting.

In humans, BH4 deficiency results in phenylalanine accumulation, which causes PKU if untreated. Over 50\% of human BH4-deficient patients suffer from PTPS deficiency [15]. The silkworm PTPS protein shares $64 \%$ amino acid identities with human PTPS. Furthermore, oral supplementation of $\mathrm{BH} 4$ and neurotransmitter precursors to silkworm $a l^{c}$ mutants rescued the defective body color, lethal phenotype, and phenylalanine metabolism, while, application of the competitive inhibitor DAHP to the wild type also effectively produced the BH4-deficient phenotype. Although the BH4 levels could not be measured directly in the above treatments due to the lack of standards, the detection of gene expressions and metabolic substances imply that silkworm alc mutant and human BH4-deficient PKU have a high similarity in the drug metabolic features and the $\mathrm{BH} 4$ biosynthesis pathway, as well as in the downstream dopamine metabolic pathway. Based on this, the $a^{c}$ mutant could be a potential disease model for human PKU.

Animal models have greatly enriched our understanding of the molecular mechanism and the therapies for disease. The silkworm is a domesticated economic insect and is one of the model organisms for basic research. It has a clear genetic background, short life span, numerous progeny, controllable living environment, easy experimental operation and feeding, mature gene editing technology, low-cost, no ethical restrictions, and a considerable number of genes that are homologous to human disease-associated genes [16]. Because of these advantages, silkworm has received increasing attention as a human disease model. Thus, many silkworm disease models have been established recently, such as Parkinson's disease [17], human sepiapterin reductase deficiency [12], insulin-related human disease [16], and Hermansky-Pudlak syndrome [18]. In addition, previous reports regarding the application of silkworm in the screening of antimicrobial drugs [19], anti-diabetic drugs, and traditional Chinese medicine [20] have implied that very similar drug metabolic features and pharmacodynamics were shared between silkworm and mammals. In the present study, the epidermis color of the $a^{c}$ mutants changed obviously upon drugs treatment. Thus, the readily visible color variation is helpful to evaluate the effectiveness of novel PKU therapeutic drug candidates. In conclusion, we presented experimental evidence that silkworm the $a l^{c}$ mutant has great potential as a suitable disease model for human BH4-deficient PKU.

\section{Materials and Methods}

\subsection{Silkworm Strains}

The $a l^{c}$ mutant and wild type strains, Dazao and N4, were supplied by the silkworm gene bank in Southwest University, China. These were reared on fresh mulberry at $25^{\circ} \mathrm{C}$ under $12 \mathrm{~h}$ light: $12 \mathrm{~h}$ dark. Second instar larvae were used unless otherwise mentioned.

\subsection{DNA Extraction and Sample Preparation}

Total genomic DNA of the parental strains and $F_{1}$ individuals was extracted from the whole moth. Genomic DNA of individuals used for mapping was extracted from whole larvae at the beginning of second instar using DNAzol ${ }^{\circledR}$ (Invitrogen, Carlsbad, CA, USA). Total RNA was isolated from Dazao and $a l^{\mathcal{C}}$ at day 0 of the second instar using TRIzol ${ }^{\circledR}$ (Invitrogen), according to the manufacturer's protocol. cDNA was synthesized from total RNA samples using random primers (N9), oligo (dT) primers, and a moloney murine leukaemia virus reverse transcriptase (Promega, Madison, WI, USA), according to the manufacturer's instructions.

\subsection{Fine Mapping of Albino C Locus}

Two silkworm strains, Dazao $(+/+)$ and albino $\mathrm{C}\left(\mathrm{al}^{\mathcal{c}} / \mathrm{al}^{c}\right)$ were used for mapping. Owing to the lack of recombination in female silkworms and the lethality of the albino $C$ mutant, we selected $\mathrm{F}_{1}$ offspring from a cross between albino $\mathrm{C}\left(+/ \mathrm{al}^{c}\right)$ o and Dazao $(+/+) \sigma^{\pi}$. Ten Dazao and ten albino C 
mutants of $\mathrm{BC}_{1} \mathrm{~F}$ progeny from the cross $\mathrm{F}_{1} q \times a l^{\mathcal{C}}\left(+/ \mathrm{al}^{\mathcal{C}}\right) \mathrm{o}^{\lambda}$ were used for linkage analysis, and 170 $\mathrm{BC}_{1} \mathrm{M}$ progeny (only $a l^{c}$ individuals) from the cross of albino $\mathrm{C}\left(+/ a l^{c}\right) \propto \times \mathrm{F}_{1}\left(+/ a l^{c}\right) 0^{\top}$ were used for recombination analysis. For fine mapping, 17 polymorphic PCR markers on chromosome 5 identified between Dazao $(+/+)$ and albino $\mathrm{C}\left(\mathrm{al}^{\mathcal{C}} / \mathrm{al}^{\mathrm{c}}\right)$ were first confirmed to be linked with the $a l^{c}$ locus by using the $\mathrm{BC}_{1} \mathrm{~F}$ progeny, and subsequently were used for recombination analysis in $\mathrm{BC}_{1} \mathrm{M}$ individuals. The molecular markers used in the linkage and mapping analysis are listed in Supplementary Table S2. This analysis required the silkworm $9 \times$ assembly genome database (http:/ /www.silkdb.org/silkdb/, SilkDB) and BLAST (http:/ / www.ncbi.nlm.nih.gov/).

\subsection{Cloning of the PTPS Gene}

Total RNA was extracted from Dazao and $a l^{c}$ at the beginning of the second instar as earlier described. The primers for the full-length cDNA and genome of the candidate genes were designed according to the sequence in GenBank and silkworm genome database (SilkDB). PCR products were cloned into the PMD19-T vector (TaKaRa, Otsu, Japan) and then sequenced. The primers used for cloning are listed in Supplementary Table S2.

\subsection{Quantitative RT-PCR Analysis}

To identify differences in the BH4 synthesis-related gene and melanin metabolism-related genes between Dazao and $\mathrm{al}^{c}$ mutant strains, total RNA was isolated using TRIzol ${ }^{\circledR}$ (Invitrogen) from the second instar larva, and then reverse transcribed to cDNA. Quantitative RT-PCR was performed using the ABI Prism 7000 sequence detection system (Applied Biosystems, Forster City, CA, USA) with a SYBR Premix EX-Taq-kit (TaKaRa, Otsu, Japan) according to the manufacturer's protocol. The primers for the GTPCH (AB439287), PTPS (KR703273), SPR (AK385894.1), DHFR (JQ348842.1), PAH (GU953670), and TH (AB439286) genes are listed in Supplementary Table S2. sw22934, a eukaryotic translation initiation factor 4A (DQ443290.1) of silkworm, was used as an internal control.

\subsection{Chemicals}

DOPA (D9628-5G), dopamine (H8502-5G), DAHP (D19206-25G), and BH4 (T4425-5 mG) were purchased from Sigma-Aldrich (St. Louis, MO, USA). The Phe and Tyr standards used for quantitative analysis were provided by the Key Laboratory in Sichuan Province, Institute of Animal Nutrition, and Sichuan Agricultural University, YaAn 625014, China.

\subsection{Quantification of Amino Acids and Catecholamine}

We selected the second instar at day 0 between wild type Dazao and alc mutant strains for amino acid and catecholamine content analysis. The free amino acids were extracted as follows: the samples were homogenized with $800 \mu \mathrm{L} 0.1 \mathrm{M}$ hydrochloric acid $(\mathrm{HCl})$ in centrifuge tubes, then the homogenate was mixed for $15 \mathrm{~min}$ using ultrasonication followed by centrifugation $\left(12,000 \times g, 4{ }^{\circ} \mathrm{C}\right)$. The supernatant $(600 \mu \mathrm{L})$ was transferred to a new tube containing $600 \mu \mathrm{L}$ of $10 \%$ sulfosalicylic acid, followed by further centrifugation $\left(12,000 \times \mathrm{g}, 4^{\circ} \mathrm{C}\right.$ for $\left.15 \mathrm{~min}\right)$. The supernatants were transferred and filtered with $0.22-\mu \mathrm{m}$ membranes. A Hitachi (Tokyo, Japan) L-8800 amino acid analyzer physiological fluid system (lithium system) was used for amino acid content analysis. DOPA and dopamine were extracted and quantified according to Koch's method [21]. Agilent1260 Infinity HPLC (Santa Clara, CA, USA) and Symmetry Shield RP18 ( $5 \mu \mathrm{M}, 4.6 \times 250 \mathrm{~mm}$, Waters Corp., Milford, MA, USA) columns were used for HPLC analysis. Compared to known standards, amino acids and catecholamine standards were identified based on retention times, as follows: Phe $56.33 \mathrm{~min}$; Tyr, $53.35 \mathrm{~min}$; DOPA $5.858 \mathrm{~min}$; and Dopamine 8.226 min (Supplementary Figure S2A,B). 


\subsection{DAHP Feeding Experiments}

DAHP (Sigma-Aldrich), an inhibitor of GTPCH, was fed to newly hatched wild type strain N4 larvae. Treatment group: $2.5 \mathrm{~g}$ of DAHP was dissolved in $0.1 \mathrm{M}$ sodium hydroxide $(\mathrm{NaOH})$, and the volume was adjusted to $150 \mathrm{~mL}$. Artificial feed $(50 \mathrm{~g})$ was then added to the $150-\mathrm{mL}$ DAHP solution, which was mixed uniformly. The mixed artificial feed was sterilized at high pressure $\left(98^{\circ} \mathrm{C}, 25 \mathrm{~min}\right)$. Control group: $50 \mathrm{~g}$ of artificial feed was added to $150 \mathrm{~mL}$ of $0.1 \mathrm{M} \mathrm{NaOH}$. The solution was then mixed until fully uniform and sterilized at high pressure $\left(98^{\circ} \mathrm{C} 25 \mathrm{~min}\right)$. The treatment was administered orally to larvae once a day for three days.

\subsection{BH4 Feeding Experiments}

BH4 (30 mM) was evenly spread on the surface of the artificial diet and fed to the newly hatched larvae once every day. The $\mathrm{F}_{2}$ offspring are an inbred line of $\mathrm{F}_{1}\left(+/ a l^{c}\right)$ (Theoretically, only $1 / 4$ larvae were $a l^{c}$ individuals from a batch). At the beginning of the second instar larvae, the alc individuals were selected for $\mathrm{BH} 4$ feeding, and then the phenotypes were observed at the second instar on day 2 . $\mathrm{BH} 4$ was administered orally once a day for three days. $\mathrm{H}_{2} \mathrm{O}$ was evenly spread on the surface of the artificial diet and was fed once a day for three days to newly hatched larvae as a control.

Supplementary Materials: Supplementary materials can be found at http:/ / www.mdpi.com/1422-0067/19/4/ $1024 / s 1$.

Acknowledgments: The Hi-Tech Research and Development 863 Program of China Grant (Grant No. 2013AA102507), the National Natural Science Foundation of China (Grant No. 31372379 and No. 31472153), and the Fundamental Research Funds for the Central Universities in China (No. XDJK2013A001) supported this study.

Author Contributions: Fangyin Dai and Cheng Lu designed the project. Hai Hu reared the silkworms. Xiaoling Tong, Pingfeng Liang, Songyuan Wu, Yuanhao Li, and Liang Qiao performed the experiments. Xiaoling Tong and Songyuan $\mathrm{Wu}$ analyzed data and drafted the manuscript. Fangyin Dai, Xiaoling Tong, Zhonghuai Xiang, and Cheng Lu supervised and revised the manuscript. All authors read and approved the final version of the manuscript.

Conflicts of Interest: The authors declare no conflict of interest.

\section{Abbreviations}

$\begin{array}{ll}\text { PKU } & \text { phenylketonuria } \\ \text { PAH } & \text { phenylalanine hydroxylase } \\ \text { BH4 } & \text { tetrahydrobiopterin } \\ \text { PTPS } & \text { 6-pyruvoyl-tetrahydropterin synthase } \\ \text { SilkDB } & \text { Silkworm Genome Database } \\ \text { GTPCH } & \text { guanosine triphosphate cyclohydrolase I } \\ \text { SPR } & \text { sepiapterin reductase } \\ \text { DHFR } & \text { dihydrofolate reductase } \\ \text { NADA } & N \text {-acetyl dopamine } \\ \text { NBAD } & N \text { - } \beta \text {-alanyldopamine } \\ \text { DAHP } & \text { hydroxypyrimidine }\end{array}$

\section{References}

1. Blau, N.; van Spronsen, F.J.; Levy, H.L. Phenylketonuria. Lancet 2010, 376, 1417-1427. [CrossRef]

2. Blau, N.; Shen, N.; Carducci, C. Molecular genetics and diagnosis of phenylketonuria: State of the art. Exp. Rev. Mol. Diagn. 2014, 14, 655-671. [CrossRef] [PubMed]

3. Berry, S.A.; Brown, C.; Grant, M.; Greene, C.L.; Jurecki, E.; Koch, J.; Moseley, K.; Suter, R.; van Calcar, S.C.; Wiles, J.; et al. Newborn screening 50 years later: Access issues faced by adults with PKU. Genet. Med. Off. J. Am. Coll. Med. Genet. 2013, 15, 591-599. [CrossRef] [PubMed]

4. Guthrie, R.; Susi, A. A Simple Phenylalanine Method for Detecting Phenylketonuria in Large Populations of Newborn Infants. Pediatrics 1963, 32, 338-343. [PubMed] 
5. Christ, S.E.; Moffitt, A.J.; Peck, D.; White, D.A. The effects of tetrahydrobiopterin (BH4) treatment on brain function in individuals with phenylketonuria. Neuroimage Clin. 2013, 3, 539-547. [CrossRef] [PubMed]

6. Heine, C.L.; Kolesnik, B.; Schmidt, R.; Werner, E.R.; Mayer, B.; Gorren, A.C. Interaction between neuronal nitric-oxide synthase and tetrahydrobiopterin revisited: Studies on the nature and mechanism of tight pterin binding. Biochemistry 2014, 53, 1284-1295. [CrossRef] [PubMed]

7. Martinez, A.; Knappskog, P.M.; Haavik, J. A structural approach into human tryptophan hydroxylase and its implications for the regulation of serotonin biosynthesis. Curr. Med. Chem. 2001, 8, 1077-1091. [CrossRef] [PubMed]

8. Thony, B.; Calvo, A.C.; Scherer, T.; Svebak, R.M.; Haavik, J.; Blau, N.; Martinez, A. Tetrahydrobiopterin shows chaperone activity for tyrosine hydroxylase. J. Neurochem. 2008, 106, 672-681. [CrossRef] [PubMed]

9. Xia, Q.; Zhou, Z.; Lu, C.; Cheng, D.; Dai, F.; Li, B.; Zhao, P.; Zha, X.; Cheng, T.; Chai, C.; et al. A draft sequence for the genome of the domesticated silkworm (Bombyx mori). Science 2004, 306, 1937-1940. [PubMed]

10. Goldsmith, M.R.; Shimada, T.; Abe, H. The genetics and genomics of the silkworm, Bombyx mori. Ann. Rev. Entomol. 2005, 50, 71-100. [CrossRef] [PubMed]

11. Duan, J.; Li, R.; Cheng, D.; Fan, W.; Zha, X.; Cheng, T.; Wu, Y.; Wang, J.; Mita, K.; Xiang, Z.; et al. SilkDB v2.0: A platform for silkworm (Bombyx mori) genome biology. Nucleic Acids Res. 2010, 38, D453-D456. [CrossRef] [PubMed]

12. Meng, Y.; Katsuma, S.; Daimon, T.; Banno, Y.; Uchino, K.; Sezutsu, H.; Tamura, T.; Mita, K.; Shimada, T. The silkworm mutant lemon (Lemon lethal) is a potential insect model for human sepiapterin reductase deficiency. J. Biol. Chem. 2009, 284, 11698-11705. [CrossRef] [PubMed]

13. Delgado-Esteban, M.; Almeida, A.; Medina, J.M. Tetrahydrobiopterin deficiency increases neuronal vulnerability to hypoxia. J. Neurochem. 2002, 82, 1148-1159. [CrossRef] [PubMed]

14. Mitchell, B.M.; Dorrance, A.M.; Webb, R.C. GTP cyclohydrolase 1 inhibition attenuates vasodilation and increases blood pressure in rats. Am. J. Physiol. Heart Circ. Physiol. 2003, 285, H2165-H2170. [CrossRef] [PubMed]

15. Opladen, T.; Hoffmann, G.F.; Blau, N. An international survey of patients with tetrahydrobiopterin deficiencies presenting with hyperphenylalaninaemia. J. Inherit. Metab. Dis. 2012, 35, 963-973. [CrossRef] [PubMed]

16. Zhang, Z.; Teng, X.; Chen, M.; Li, F. Orthologs of human disease associated genes and RNAi analysis of silencing insulin receptor gene in Bombyx mori. Int. J. Mol. Sci. 2014, 15, 18102-18116. [CrossRef] [PubMed]

17. Tabunoki, H.; Ono, H.; Ode, H.; Ishikawa, K.; Kawana, N.; Banno, Y.; Shimada, T.; Nakamura, Y.; Yamamoto, K.; Satoh, J.; et al. Identification of key uric acid synthesis pathway in a unique mutant silkworm Bombyx mori model of Parkinson's disease. PLoS ONE 2013, 8, e69130. [CrossRef] [PubMed]

18. Fujii, T.; Daimon, T.; Uchino, K.; Banno, Y.; Katsuma, S.; Sezutsu, H.; Tamura, T.; Shimada, T. Transgenic analysis of the BmBLOS2 gene that governs the translucency of the larval integument of the silkworm, Bombyx mori. Insect Mol. Biol. 2010, 19, 659-667. [CrossRef] [PubMed]

19. Hanada, Y.; Sekimizu, K.; Kaito, C. Silkworm apolipophorin protein inhibits Staphylococcus aureus virulence. J. Biol. Chem. 2011, 286, 39360-39369. [CrossRef] [PubMed]

20. Chen, C.; Song, J.; Chen, M.; Li, Z.; Tong, X.; Hu, H.; Xiang, Z.; Lu, C.; Dai, F. Rhodiola rosea extends lifespan and improves stress tolerance in silkworm, Bombyx mori. Biogerontology 2016, 17, 373-381. [CrossRef] [PubMed]

21. Koch, P.B.; Behnecke, B.; Weigmann-Lenz, M.; Ffrench-Constant, R.H. Insect pigmentation: Activities of $\beta$-alanyldopamine synthase in wing color patterns of wild-type and melanic mutant swallowtail butterfly Papilio glaucus. Pigment Cell Melanoma Res. 2000, 13 (Suppl. 8), 54-58. [CrossRef]

(C) 2018 by the authors. Licensee MDPI, Basel, Switzerland. This article is an open access article distributed under the terms and conditions of the Creative Commons Attribution (CC BY) license (http:// creativecommons.org/licenses/by/4.0/). 